\title{
A dynamic view of cellular processes by in vivo fluorescence auto- and cross-correlation spectroscopy
}

\author{
Kirsten Bacia and Petra Schwille* \\ Experimental Biophysics Group, Max-Planck-Institut für biophysikalische Chemie, Am Fassberg 11, D-37077 Göttingen, Germany
}

Accepted 11 September 2002

\begin{abstract}
Fluorescence correlation spectroscopy (FCS) is becoming increasingly popular as a technique that aims at complementing live cell images with biophysical information. This article provides both a short overview over recent intracellular FCS applications and a practical guide for investigators, who are seeking to integrate FCS into live cell imaging to obtain information on particle mobility, local concentrations, and molecular interactions. A brief introduction to the principles of FCS is provided, particularly emphasizing practical aspects such as the choice of appropriate dyes and positioning of the measurement volume in the sample. Possibilities and limitations in extracting parameters from autocorrelation curves are discussed, and attention is drawn to potential artifacts, such as photobleaching and probe aggregation. The principle of dual-color cross-correlation is reviewed along with considerations for proper setup and adjustment. Practical implications of nonideal conditions including incomplete focus overlap and spectral crosstalk are considered. Recent examples of both auto- and cross-correlation applications demonstrate the potential of FCS for cell biology.
\end{abstract}

(C) 2002 Elsevier Science (USA). All rights reserved.

Keywords: Fluorescence correlation spectroscopy; Single-molecule analysis; In vivo; Photobleaching; Colocalization; Confocal microscopy; Fluorescence resonance energy transfer; Fluorescence recovery after photobleaching

\section{Introduction}

The invention of the microscope in the 17th century provided the catalyst for a new field of science based on the idea that life is composed of building blocks, called cells. Each cell has the capability to propagate by division and "bears in itself the complete characteristics of life" (Rudolph Virchow, 1858 [1]). Not surprisingly, biological research today strives toward understanding the tremendously complex network of cellular processes.

While biochemical techniques have allowed for separation of cellular components, genetics and molecular biology have made identifying proteins of interest even more accessible. Isolated components or proteins can thereby be used in in vitro experiments to model biochemical reactions and molecular interactions and to reproduce complex cellular processes involving multiple components and structures. For example, binding ex-

\footnotetext{
${ }^{*}$ Corresponding author. Fax: +49-551-201-1435.

E-mail address: pschwil@gwdg.de (P. Schwille).
}

periments aim to describe interactions in terms of thermodynamic and kinetic parameters (binding and rate constants).

Prior cell biological assays worked with live cells but require post hoc analysis. For example, endocytosis can be studied by adding radiolabeled or fluorescently labeled cargos to live cells, and assaying for the markers after subcellular fractionation. However, this may lead to artifacts due to potential changes in the content of the compartments during isolation.

Biochemical experiments provide a simplified, controllable system; however, it is essential to be able to also observe and quantitate processes directly in live eukaryotic cells. Due to the cell's compartmentalized structure, subcellular localization, concentration, and mobility of the molecules of interest are as important as their mere function. Furthermore, all other potentially interacting cellular constituents are present and the native intracellular milieu is left intact.

Fluorescence is a parameter that lends itself to live cell applications due to its high specificity and the low inter- 
ference of optical detection with cell function. Confocal fluorescence laser scanning microscopy (LSM) is widely used for imaging optical sections of fixed and live samples. In addition to imaging, LSM can be used to a limited extent to estimate mobility and the effects of compartmentalization through photobleaching techniques (FRAP, FLIP, etc.; see also other articles in this issue). Furthermore, fluorescence resonance energy transfer (FRET) between two fluorophores in close proximity has been extended from in vitro experiments to in vivo applications, providing information about inter- or intramolecular distances in combination with spatial resolution.

Fluorescence correlation spectroscopy (FCS) is the latest addition to biophysical techniques used in conjunction with imaging. Based on a confocal optical setup, similar to a laser scanning microscope (Fig. 1), FCS relies on different principles than the earlier mentioned techniques and has different advantages and limitations. It is becoming increasingly evident that live cell auto- and cross-correlation can provide new insights into cellular processes previously inaccessible in vivo as is discussed here. Also, some of the pitfalls associated with the use of this technique are highlighted, and strategies to overcome some current limitations associated with in vivo FCS are mentioned.

\section{Laser scanning microscopy combined with fluorescence correlation spectroscopy}

Whereas in LSM, statistical fluctuations in the fluorescence intensity from a small detection volume, arising at low concentrations and short integration times, represent an undesired source of noise, these fluctuations constitute the signal in FCS (Fig. 2) and need to be recorded with high temporal resolution. Although higher concentrations are normally employed in LSM to improve signal quality, a concentration range can generally be found that supports both acceptable quality of visualization in LSM and good FCS fluctuation measurements. Thus, the use of a combined setup incorporating both techniques is practicable. This combination of FCS with not only classic fluorescence and transmitted light microscopy, but also confocal microscopy in a single setup is very valuable, since it allows for positioning of the FCS measurement volume with better (axial) resolution. Also, recording of the FCS measurement along with a confocal image of the object aids in interpretation of the data.

There are different possibilities for the implementation of a combined LSM and FCS system. One alternative is to have fully separate modules for LSM and FCS detection, as realized in the commercially available ConfoCor2 system by Carl Zeiss (Jena, Germany). This

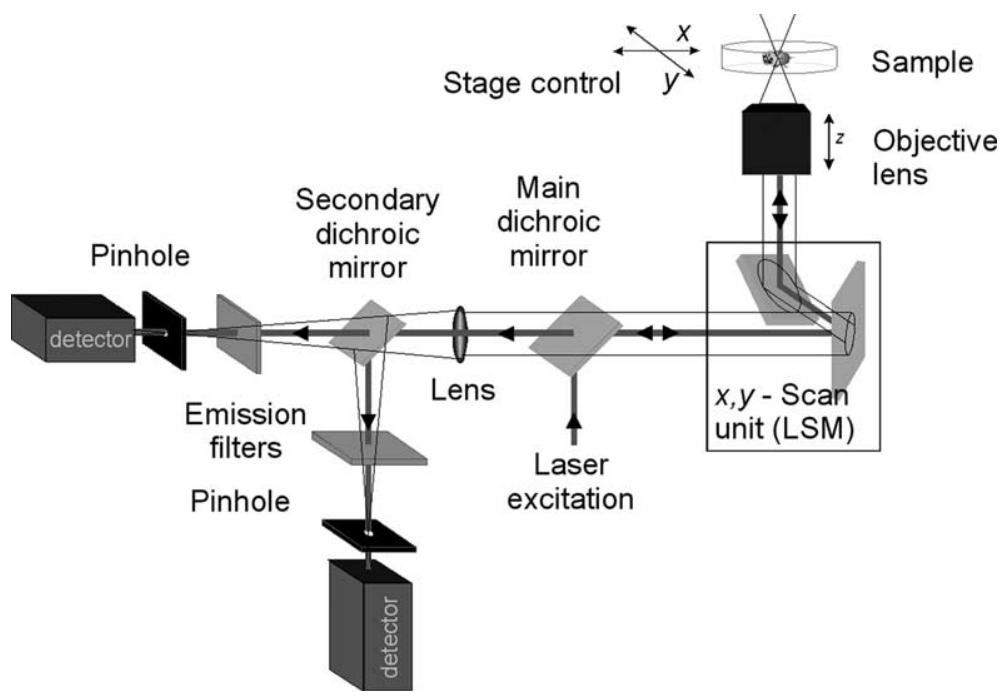

Fig. 1. Optical setup used in laser scanning microscopy (LSM) and fluorescence correlation spectroscopy (FCS). Both optical techniques use a confocal setup, usually based on an inverted microscope: The parallel laser light from one or two lasers is reflected onto the back aperture of the objective. Homogeneously illuminating the back aperture of the objective results in a diffraction-limited excitation volume ("spot") in the sample. This is mostly realized by overfilling the back aperture with a broad Gaussian beam profile. The Stokes-shifted fluorescence light from the sample passes straight through the main dichroic mirror and is focused onto a pinhole and detected by a photomultiplier tube (PMT) or avalanche photodiode (APD). The pinhole allows for "optical slicing," as light from below or above the excitation spot in the sample is rejected by the pinhole. For good axial resolution, high-NA objectives need to be employed. Since buffer solutions and live biological samples are water-like, water immersion is preferred to minimize aberrations. In the case of LSM image slice acquisition, the "spot" is scanned laterally $(x-y)$ through the sample, either by using scanning mirrors to deflect the beam or by moving the sample. In contrast, for normal FCS measurements, the FCS spot is positioned before the measurement and then kept stationary in the sample during data acquisition. For dual-color LSM or FCS, the fluorescence emissions from the two fluorophores in the sample are separated by a secondary dichroic mirror and simultaneously detected by two separate detectors ("channels"). 

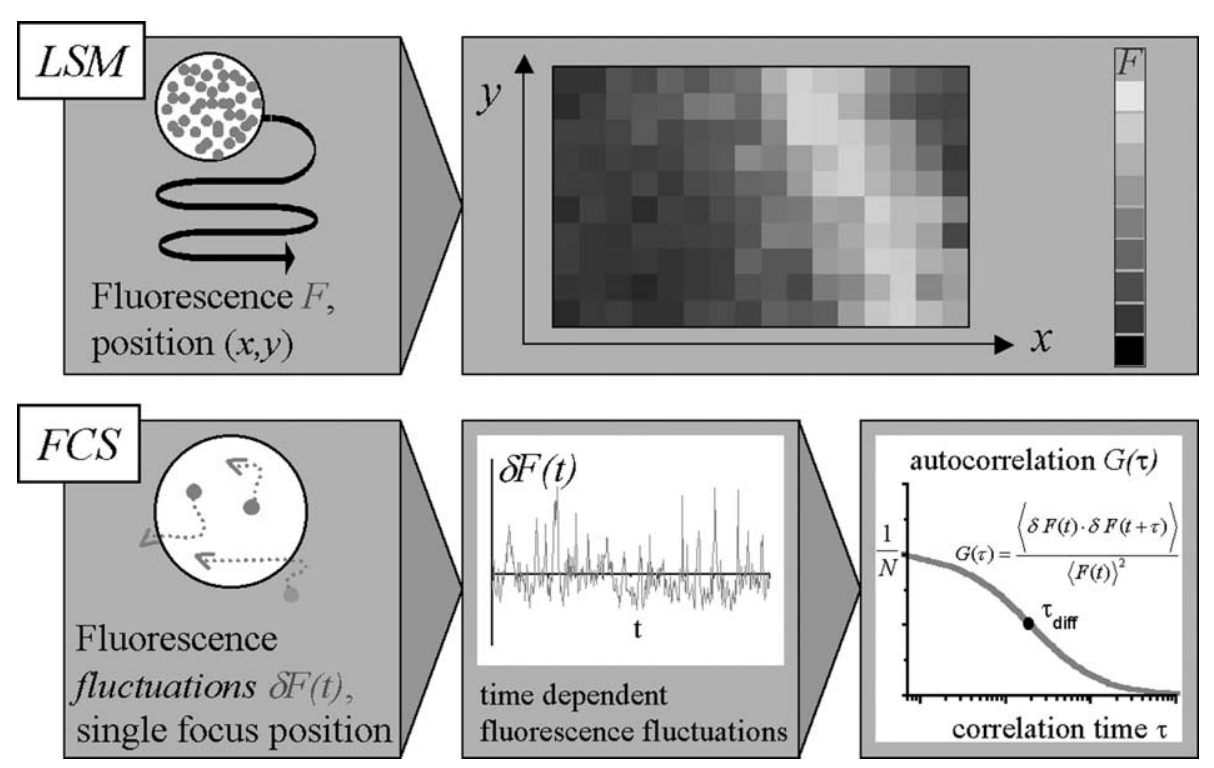

Fig. 2. Acquisition and processing of the fluorescence signal in laser scanning microscopy (LSM) and fluorescence correlation spectroscopy (FCS). In LSM, the total fluorescence from the confocal detection volume is recorded. Low fluorophore concentrations along with short integration times (high-speed scans, high-resolution scans) only lead to undesired noise due to statistical fluctuations of the number of fluorophores in the detection volume. In contrast, in FCS, the stastical fluctuations of fluorophores in the small confocal detection volume constitute the measurement signal. Thus, low concentrations and bright fluorophores are required to obtain an FCS signal. However, in in vivo measurements, fluorophore concentrations should not be too low, as the fluorescence signal needs to be considerably above the background, arising for instance from cellular autofluorescence and scattering. Depending on the size of the detection volume, the brightness of the fluorophores, and the background, the suitable range is typically from below $100 \mathrm{nM}$ to up to $1 \mu \mathrm{M}$. The fluorescence fluctuations are mathematically processed using an autocorrelation algorithm. The resulting autocorrelation curve is interpreted by fitting to equations that have been derived for different diffusion models. In simple cases, the number of fluorophores in the detection volume can be read from the reciprocal of the amplitude and the diffusion time from the half-value decay of the autocorrelation curve. From these parameters, the concentration and the diffusion coefficient of the labeled particles can be calculated, if a simple in vitro calibration measurement with a dye solution has also been performed.

setup has the advantage that it makes use of the existing highly advanced LSM technology, in which the beam is scanned in the lateral $(x y)$ direction by scanning mirrors, without the need to subject the FCS beam to a passage through these potentially perturbing optical components. Also, separate detectors can be used, generally photomultiplier tubes (PMTs) for LSM, which can tolerate high photon fluxes without deviations from linear detection characteristics, and avalanche photodiodes (APDs) for FCS, which generally provide a much higher quantum efficiency, but are easily damaged by high light intensities. The disadvantage of this kind of combined system where the LSM beam is scanned by means of mirrors, while the FCS beam is positioned through movement of the sample stage, consists of the fact that the lateral FCS beam positioning accuracy is currently limited to about $\sigma=0.3 \mu \mathrm{m}$. Other, home-built setups use only one beam and one detector (an APD) for LSM and FCS, either by $x y$-scanning the beam using mirrors [2] or $x y$-scanning the stage with a piezo drive [3].

\section{Information from fluorescence autocorrelation mea- surements}

Different methods for evaluating time-dependent fluorescence fluctuations provide access to different pa- rameters. For example, autocorrelation analysis [4-8] is suitable for investigating mobilities, whereas related methods of photon counting histograms [9-11] and similar approaches [12] analyze the fluctuations to obtain information on different brightnesses of species. Most in vivo applications so far have used correlation analysis applying one of the following definitions of the autocorrelation function:

$$
G^{\delta F}(\tau)=\langle\delta F(t) \delta F(t+\tau)\rangle /\langle F(t)\rangle^{2}
$$

or

$$
G^{F}(\tau)=\langle F(t) F(t+\tau)\rangle /\langle F(t)\rangle^{2},
$$

where \langle\rangle denotes the time average, $\delta F(t)=F(t)-\langle F(t)\rangle$ denotes the fluctuations around the mean intensity and for stable long time average of $F$ (no bleaching), $G^{\delta F}=G^{F}-1$.

Calculation of the autocorrelation curve may be performed off-line or on-line, by hardware correlator cards or by fast software computation. With online correlation, the resulting $G(\tau)$ curve is conveniently displayed during the measurement, along with a fluorescence trace with low time resolution, which allows visual inspection of the stability of the measurement, i.e., if bleaching or unexpectedly large fluctuations in the count rate are present. For a number of simple diffusion models, fluorescence autocorrelation theory [reviewed in 
$13,14]$ yields analytical functions, which are fitted to the measured curves by nonlinear least-squares fitting, a procedure implemented in a number of data analysis packages (e.g., Origin, OriginLab, Northampton, MA) and in the ConfoCor2 system software.

For example, in a system in which one fluorescent species is undergoing free isotropic Brownian diffusion in all three dimensions, the fluorescence autocorrelation curve is fitted to the function

$G(\tau)=N_{\text {eff }}^{-1}\left(1+\tau / \tau_{\text {diff }}\right)^{-1}\left(1+\tau /\left(S^{2} \tau_{\text {diff }}\right)\right)^{-1 / 2}$.

The structure parameter $S$ characterizes the shape of the ellipsoidal detection volume (quotient of axial and lateral radius, $S=z_{o} / \omega_{o}$ ). The diffusion time, i.e., the average lateral transit time of the particles through the focus, $\tau_{\text {diff, }}$ relates to the diffusion coefficient by $D=\omega_{o}^{2} / 4 \tau_{\text {diff }} . N_{\text {eff }}$ denotes the average number of particles residing in the effective measurement volume, $V_{\text {eff }}=\pi^{3 / 2} S \omega_{o}^{3}$. Consequently, $\omega_{o}, S$, and $V_{\text {eff }}$ can be determined from a simple calibration measurement with a solution of freely diffusing particles of known diffusion properties, which then allow for calculation of diffusion coefficients $D$ from characteristic diffusion times $\tau_{\text {diff }}$, and of local concentrations from particle numbers $N_{\text {eff }}$. For large structure parameters $S$ or two-dimensional diffusion, as expected in planar membranes, the equation simplifies to

$G(\tau)=N_{\text {eff }}^{-1}\left(1+\tau / \tau_{\text {diff }}\right)^{-1}$.

Fluorescence fluctuations arise not only from diffusion through the focal volume, but also from reversible transitions into nonfluorescent states on a faster time scale (triplet transition [15], isomerization [16], reversible protonation [17], etc.) Therefore, it is usually necessary to include one or more exponential terms in the fitting function, as shown in Fig. 3.

\section{Dynamic range, photobleaching}

Fluorescence correlation spectroscopy commonly accesses dynamics on time ranges between $1 \mu \mathrm{s}$ and $100 \mathrm{~ms}$. For convenient plotting of this very wide dynamic range, a logarithmic time scale is generally used (Fig. 4). When analyzing dynamics on the very fast scale (faster than the translational diffusion of small molecules through a diffraction-limited focus, i.e., for instance triplet blinking dynamics or rotational diffusion), special precautions need to be taken to avoid artifacts. For example artifacts arising from detector afterpulsing depend on the kind of detector being used and can be prevented by splitting the emission light in half, directing it onto two detectors, and computing the crosscorrelation function rather than the autocorrelation functions.

Since FCS temporal resolution is below $100 \mathrm{~ns}$, limitations of FCS for in vivo applications will more likely appear in the slow time range. When analyzing slowly moving molecules, e.g., molecules interacting with the cytoskeleton, nuclear DNA, or membranes, one has to ensure that acquisition times are sufficiently long to capture the process and, most importantly, that the molecules under investigation are not photobleached during their transit time through the focal volume. Photobleaching causes an erroneously fast diffusion (since the bleaching of the molecules mimics exit from the focal volume) and the photobleaching decay in the fluorescence trace leads to a decay in the correlation

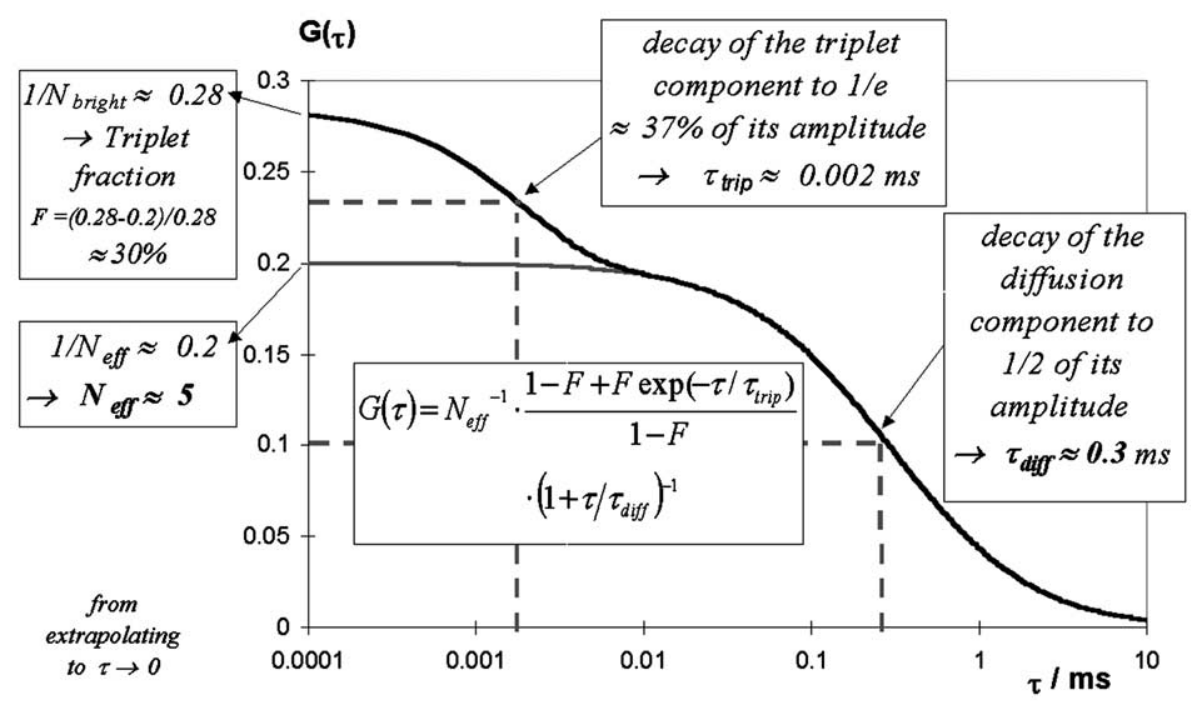

Fig. 3. Parameters in a simple autocorrelation curve. Computed fluorescence correlation curve for two-dimensional diffusion including triplet blinking. Evidently, the reciprocal of the number of particles in the detection volume determines the amplitude of the FCS curve; the kinetics of the triplet blinking process (relaxation time $\tau_{\text {trip }}$ ) and the particle mobility (diffusion time $\tau_{\text {diff }}$ ) determine the two characteristic decays in this curve. 
curve (Fig. 3e in [18]) which often prevents proper curve evaluation by standard models. With the software corrrelation implemented in the ConfoCor2 system, this

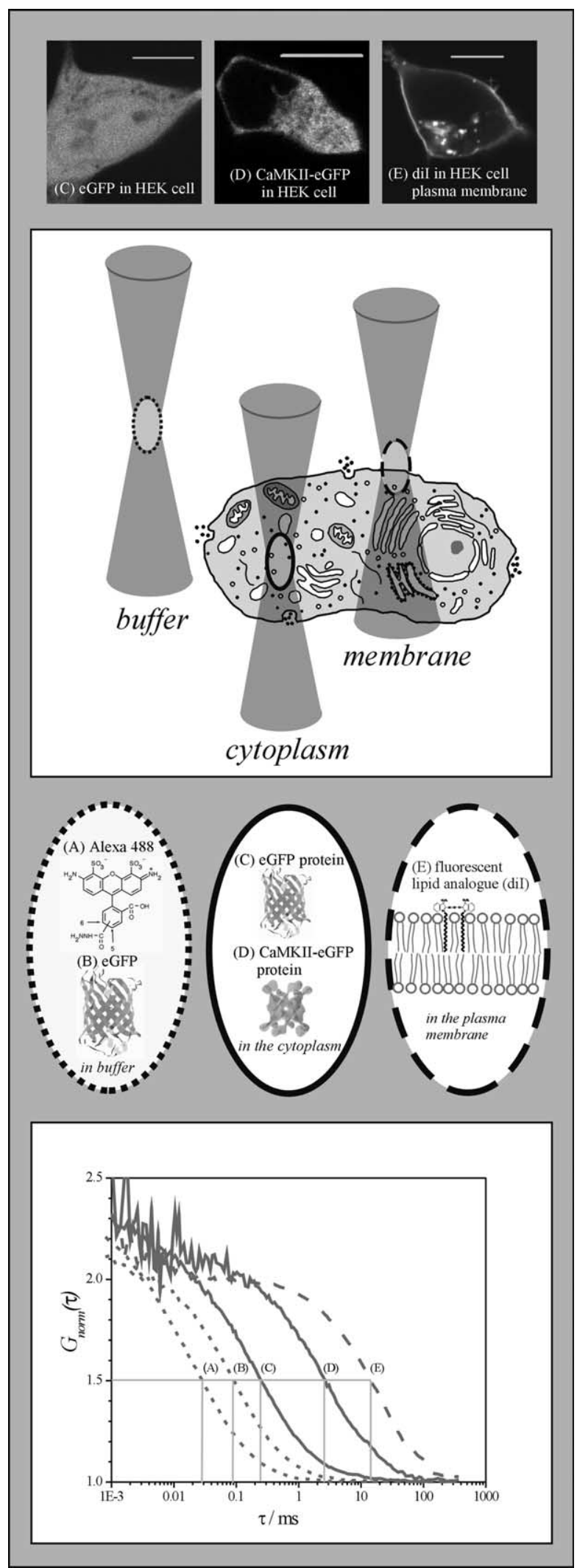

decay is characterized by a drop of the curve below the $G(\tau)=1$ line, but may appear differently depending on the normalization scheme used by the correlator. Thus, it is rather important to check the influence of the laser power on the fluorescence trace and the correlation curves before recording the actual measurements. Also, one should be aware that the molecule of interest is likely to be less mobile and thus more easily bleached in the live cell than in vitro. In particular, a heterogeneity of diffusing species might be observed in vivo, exhibiting a distribution of mobilities. Thus, if the laser is allowed to illuminate the sample before the start of the data acquisition, a potential immobile fraction can be bleached away. This prebleach is inevitable if the stage is positioned during observation of the fluorescent spot produced by the laser beam in the sample, but it can be avoided if the positioning is carried out "blindly" according to a scanned image. (Photobleaching during scanning is not biased toward less mobile molecules.) However, the prebleach may also deliberately be used to be able to observe the mobile fraction afterward (Fig. 5 in [18]). Particularly for the slow diffusion encountered in membrane applications, it may be hoped that fluorophores with even higher photostability will lead to an extension of the accessible time range. In the case of dual-color cross-correlation analysis, which is discussed later, another resort from the immobility problem is "scanning FCS" [3,19], where the motion of the particles is replaced by the motion of the focus relative to the sample, thus giving up any mobility information.

\section{Suitable dyes}

Whereas in fluorescence imaging, bleaching might easily be avoided by working at lower laser intensities or shorter acquisition times and simply increasing probe concentration, this is not a solution when doing FCS. In FCS, the signal-to-noise ratio is governed largely by the

Fig. 4. Autocorrelation measurements assess a wide span of dynamic behavior in vitro and in vivo. The FCS focal volume, which is approximately $0.2 \mathrm{fl}$, can be positioned, e.g., in the cytoplasm, the nucleus, the plasma membrane of a cell, or outside the cell. (For membrane measurements, the focus is positioned at the maximum count rate or brightness obtained in an axial scan through the labeled upper or lower membrane.) Depending on the probe and the environment, diffusional mobilities on different orders of magnitude are obtained: (A) small dye molecule (Alexa $488, \approx 0.6 \mathrm{kDa}$ ) in water, $\tau_{\text {diff }}=0.029 \mathrm{~ms}, D=3 \times 10^{-6} \mathrm{~cm}^{2} / \mathrm{s}$; (B) purified protein (eGFP, $\approx 27 \mathrm{kDa})$ in aqueous solution, $\tau_{\text {diff }}=0.090 \mathrm{~ms}, D=9 \times 10^{-7} \mathrm{~cm}^{2} / \mathrm{s}$; (C) eGFP protein in the cytoplasm of a HEK cell, $\tau_{\text {diff }}=0.27 \mathrm{~ms}$, $D=3 \times 10^{-7} \mathrm{~cm}^{2} / \mathrm{s}$; (D) large protein complex (multimeric complex of a calmodulin-dependent kinase II-eGFP fusion protein) in the cytoplasm of a HEK cell, $\tau_{\text {diff }}=2.6 \mathrm{~ms}, D=3 \times 10^{-8} \mathrm{~cm}^{2} / \mathrm{s}$; (E) two-dimensional diffusion of a fluorescent lipid analog (long-chain carbocyanine dye "diI $C_{18}$ ") in the plasma membrane of a HEK cell, $\tau_{\text {diff }}=14 \mathrm{~ms}, D=6 \times 10^{-9} \mathrm{~cm}^{2} / \mathrm{s}$. Bars $=10 \mu \mathrm{m}$. 
molecular brightness. Therefore, the setup alignment always needs to be optimized (daily adjustment of the pinholes is recommendable) and the fluorophores need to be selected for excellent absorbance, quantum yield, and photostability before being used in an FCS application. Experimentally, these properties are summarized by the brightness parameter $\eta$ ("counts per molecule" or $\mathrm{cpm}$, in units of $\mathrm{kHz}$ ), describing the count rate per particle that can be achieved. This parameter is readily calculated as the quotient of the average fluorescence count rate in the measurement and the number of particles in the detection volume, as determined from the correlation curve (Fig. 3).

\subsection{Chemical labeling}

Among the dyes that can be covalently linked to biological molecules via reactive groups, a substantial number have proven useful for FCS experiments. Suitable examples include rhodamine dyes (rhodamine green, tetramethylrhodamine, rhodamine 6G), cyanine dyes (Cy2, Cy3, Cy5, from Amersham Biosciences, Piscataway, NJ; diO, diI, diD from Molecular Probes, Eugene, OR), Bodipy and Alexa dyes (e.g., Bodipy 630/ 650, Alexa 488, Alexa 546, from Molecular Probes), but not, for instance, fluorescein. The Alexa dyes mentioned may be preferable over the rhodamine dyes for in vivo applications, because they exhibit a lower hydrophobicity [20]. Since in FCS, the fluorescence properties of the single diffusing units are of crucial relevance, the signal is considerably improved by a high labeling ratio, i.e., if one looks at the diffusion of beads, vesicles, or proteins loaded with several dye molecules each. On the other hand, an excessively high labeling ratio may interfere with biological function.

\subsection{Effect of inhomogeneous brightnesses}

Furthermore, if the labeling is random, there will be species of different brightness. If the brightness distribution function is unknown, this uncertainty impedes correct interpretation of particle numbers (concentrations): Suppose there are two species of different brightness, $\eta_{\mathrm{A}}$ and $\eta_{\mathrm{B}}$, then the apparent number of molecules in the detection volume will be lower than the total number of molecules but higher than the number of brighter (better visible) molecules. For example, according to Eq. (4), if half of the molecules $\left(X_{\mathrm{A}}=X_{\mathrm{B}}=0.5\right)$ are twice as bright $\left(\eta_{\mathrm{B}}=2 \eta_{\mathrm{A}}\right)$ as the other half, there appear to be $10 \%$ less molecules overall.

Random labeling of proteins is usually done by aminoreactive labeling procedures. A distinct labeling ratio can usually be achieved in nucleic acid labeling. In protein labeling, distinct labeling ratios of one fluorophore per subunit are achieved if the protein is mutated to have exactly one Cys residue and a thiol-reactive probe is used.
Another option for one-to-one labeling of proteins is the cloning of a fusion construct with a fluorescent protein.

\subsection{Use of fluorescent proteins in FCS}

Fluorescent proteins are very convenient for in vivo FCS. eGFP and YFP (available from Clontech, Palo Alto, CA; for a review see [21]) provide excellent properties for FCS. CFP seems less suited due to its lower brightness and higher susceptibility to photobleaching. In addition to being a bright and photostable chromophore, eGFP shows a blinking process in the range 10$100 \mu \mathrm{s}$, which is due to a reversible protonation of tyrosine-66 residue in the chromophore [17] and could in principle be used as an in vivo $\mathrm{pH}$ indicator. As opposed to eGFP, YFP additionally shows a strong excitation intensity-dependent blinking process in this time range [22], which does not interfere with in vivo applications provided the diffusion of the construct is significantly slower than the blinking. The red fluorescent protein DsRed also shows intensity-dependent blinking processes which have been investigated in detail by FCS [23]. Unfortunately, basically all red fluorescent proteins that are commercially available so far (DsRed, DsRed2, hcRed) show a tendency to form oligomers or aggregates. Nevertheless, there is a true need for red fluorescent proteins to be combined with eGFP for dual-color applications [24]; thus it should be hoped that red fluorescent proteins with better properties become available soon. Recently, a new mutant of DsRed, mRFP1 (monomeric red fluorescent protein [25]) has been reported, but from its extinction coefficient, quantum yield and photobleaching rates, there is not much hope that it is the much sought-after red probe.

\section{Two-photon excitation}

Denk et al. $[26,27]$ first demonstrated that two-photon excitation is an elegant solution to obtain intrinsic $3 \mathrm{D}$ resolution in LSM, with the additional advantage that photodamage is confined to the immediate vicinity of the focal plane. By applying two-photon excitation to intracellular FCS [28], it could indeed be verified that in comparison to conventional single-photon FCS, twophoton excitation at the same signal levels minimizes photobleaching in spatially restrictive cellular compartments. The advantages already known from imaging applications, such as reduced scattering and higher penetration depths in turbid tissues, could also be confirmed. Two-photon excitation requires the absorption of two photons of approximately double the wavelength as usual within a very short time interval $\left(10^{-15} \mathrm{~s}\right)$. To obtain a reasonable probability for this event, the instantaneous photon flux must be extremely high. This is achieved by using high output power and pulsed (best: 
femtosecond) laser systems, which are unfortunately more costly than lasers used for conventional singlephoton excitation. Since the two-photon-induced transition to the excited state, which is formally symmetry forbidden, exhibits different selection rules and vibronic coupling, it is possible to accomplish reasonable excitation of spectrally distinct dyes with a single IR laser line in selected dye systems, making two-photon excitation ideal for cross-correlation schemes [29].

\section{Binding studies by autocorrelation}

\subsection{Reliability of multicomponent fitting}

The large time range accessible by FCS makes it possible to analyze the superposition of various diffusion (and/or blinking) processes that take place on different time scales in a single FCS measurement (Fig. 5). Their respective time scales can be revealed by fitting the au- tocorrelation curve to a multicomponent diffusion model. For instance, the model for two components with fractions $X_{\mathrm{A}}$ and $X_{\mathrm{B}}$, brightness values $\eta_{\mathrm{A}}$ and $\eta_{\mathrm{B}}$, and diffusion times $\tau_{\mathrm{A}}$ and $\tau_{\mathrm{B}}$ reads

$$
\begin{aligned}
G(\tau)= & N_{\text {eff }}^{-1}\left\{Y_{\mathrm{A}}\left(1+\tau / \tau_{\mathrm{A}}\right)^{-1}\left(1+\tau /\left(S^{2} \tau_{\mathrm{A}}\right)\right)^{-1 / 2}\right. \\
& \left.+Y_{\mathrm{B}}\left(1+\tau / \tau_{\mathrm{B}}\right)^{-1}\left(1+\tau /\left(S^{2} \tau_{\mathrm{B}}\right)\right)^{-1 / 2}\right\},
\end{aligned}
$$

with $Y_{\mathrm{A}}=\eta_{\mathrm{A}}^{2} X_{\mathrm{A}} /\left(\eta_{\mathrm{A}} X_{\mathrm{A}}+\eta_{\mathrm{B}} X_{\mathrm{B}}\right)^{2}, Y_{\mathrm{B}}$ is analogous.

If - and only if - the two species exhibit equal brightness, $\eta_{\mathrm{A}}=\eta_{\mathrm{B}}, Y_{\mathrm{A}}$ and $Y_{\mathrm{B}}$ simply represent their fractions $X_{\mathrm{A}}$ and $X_{\mathrm{B}}$.

Due, however, to the nature of the hyperbolic decay of the obtained correlation curves, the diffusion of multiple species can be resolved only if their diffusion times are well separated. Computer simulations by Meseth et al. [30] show that for good signal quality this could in principle be achieved if $\tau_{\mathrm{A}} / \tau_{\mathrm{B}}$ equals at least 1.6 , but for worse signal and more disparate fractions, a two-component fit may not be justified even for $\tau_{\mathrm{A}} / \tau_{\mathrm{B}}=10\left(Y_{\mathrm{B}}=0.1 \mathrm{Fig}\right.$. 6 in
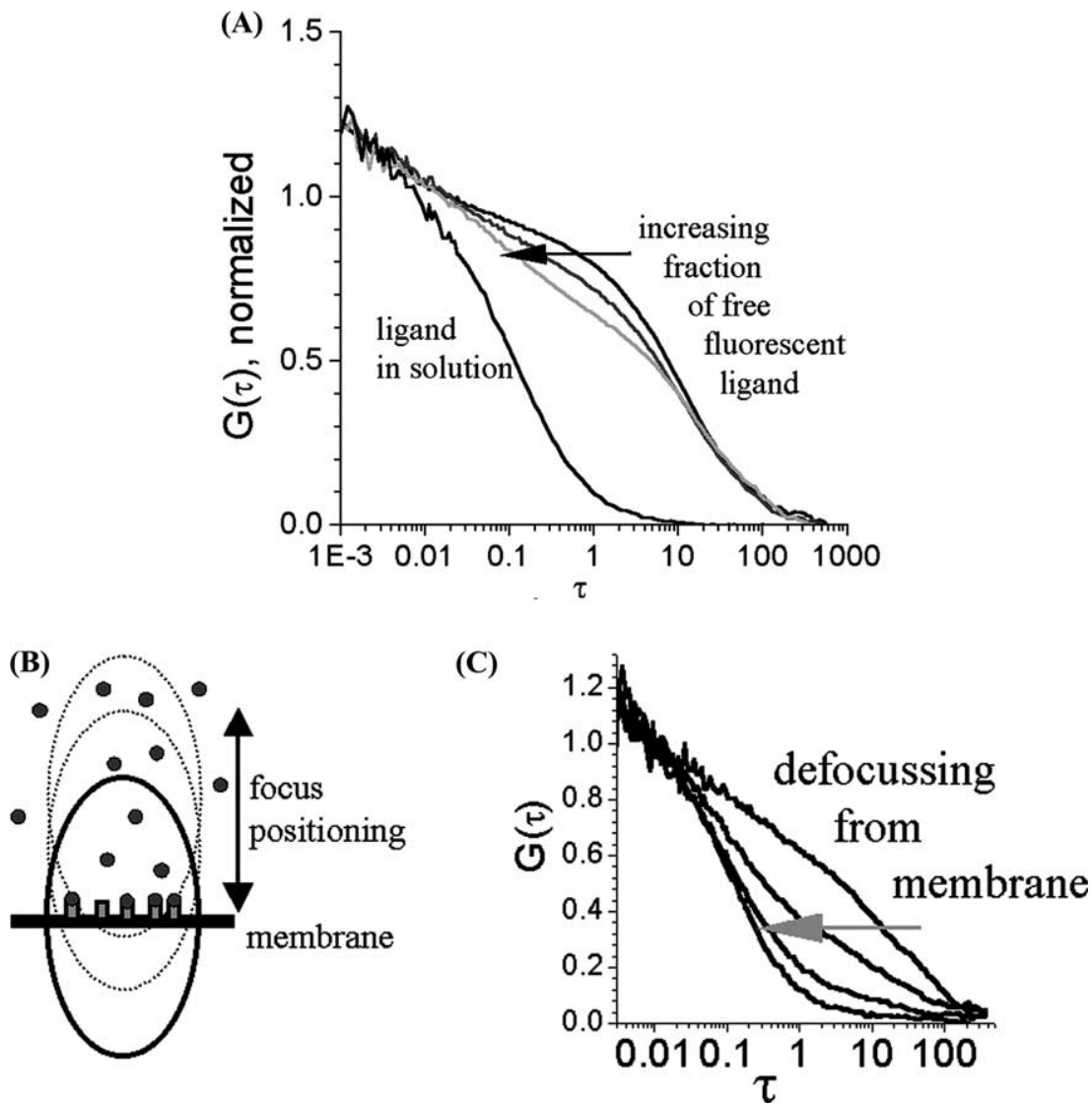

Fig. 5. Multicomponent fitting. FCS measurements on an artificial membrane [giant unilamellar vesicle (GUV)] that contains a nonlabeled receptor (ganglioside GM1). Fluorescently labeled ligand (cholera toxin B subunit, labeled with Alexa 488) has been added at different concentrations. Note that in this experiment, the membrane-bound ligand diffuses about two orders of magnitude slower than the free ligand in solution $\left(D=5 \times 10^{-7} \mathrm{~cm}^{2} / \mathrm{s}\right)$. Therefore, the two components are quite easily discernable. At low ligand concentrations, the diffusion curve is governed by the membrane-bound fraction. At higher concentrations, there is an increasing fraction of free ligand in the focus. The fractions of free and bound ligand are sensitive to the positioning of the focus on the membrane: When the focus is positioned above the membrane as depicted in (B), more fastdiffusing free ligand contributes to the FCS curve $(C)$. The FCS curves in $(C)$ were recorded at $0.3-\mu \mathrm{m}$ focus steps in the axial direction. To alleviate the problem of positioning, the application of cross-correlation spectroscopy appears very promising. 
(A)

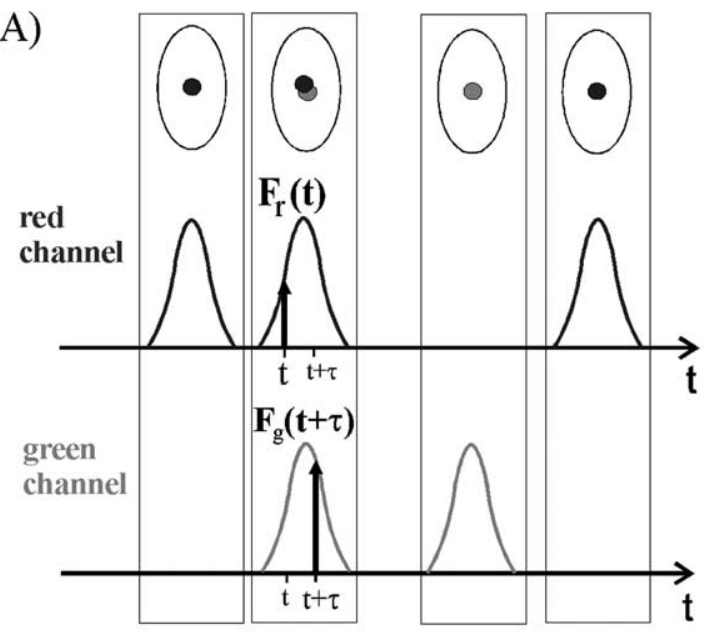

(B)

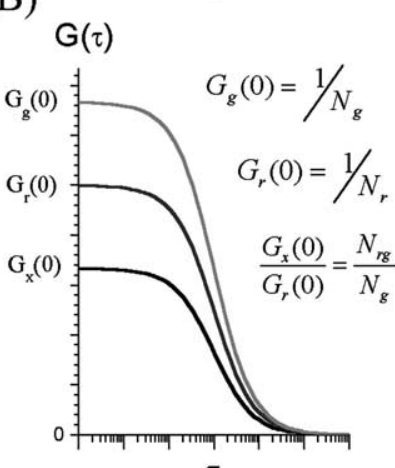

$\tau$

$$
G_{x}(\tau)=\frac{\left\langle\delta F_{r}(t) \delta F_{g}(t+\tau)\right\rangle}{\left\langle F_{r}(t)\right\rangle\left\langle F_{g}(t)\right\rangle}
$$

(C)

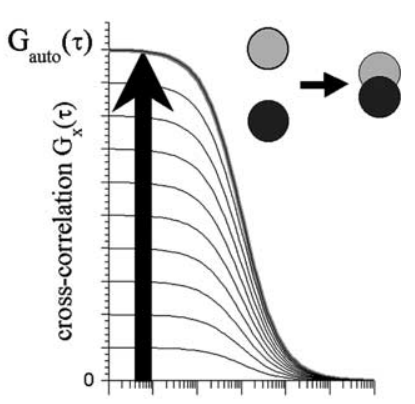

$\tau$

Fig. 6. Cross-correlation in an idealized setup. (A) The signals in the red and green channels arise from red-, green-, and double-labeled particles diffusing through the focus. All particles carrying red labels, including the double-labeled ones, appear in the red channel and therefore contribute to the red autocorrelation curve (B); green labeled-particles, including those carrying both labels, contribute to the green autocorrelation curve. Only double-labeled particles contribute to the cross-correlation amplitude. The cross-correlation amplitude relates to, for instance, the green autocorrelation amplitude as the number of double-labeled particles relates to the total number of particles carrying red labels. (C) The greater the fraction of double-labeled particles, the higher the cross-correlation amplitude relative to the autocorrelation amplitudes.

[30]). Care has to be taken in interpreting results from multicomponent fits, since invoking too many parameters may seemingly improve the fit without providing reliable parameter values. When fitting a two-component diffusion in the case where one of the components exists only in a low fraction, the fit results for these fraction parameters are often biased toward values closer to each other than the expected values. Since some parameters in the model equations are strongly interdependent (correlated), it is helpful if calibration and control measurements are done and as many parameters are fixed as possible. Usually, the structure parameter, which describes the shape (elonga-

tion) of the detection volume and is interdependent with the diffusion time, needs to be determined beforehand in an in vitro calibration measurement of free dye. Also, if each species can be measured separately, diffusion times can be fixed in a multicomponent fitting procedure.

\subsection{Effect of singular bright events on fluorescence correlation curves}

Special attention should also be devoted to the possibility of singular very bright events (which may result from aggregates). Since the brightness contributes as $\eta$ squared to the numerator in Eq. (4), a singular bright diffusion event ("single transition event" [2]), which may be recognizable from visual inspection of the fluorescence trace, can appear as a significant or even dominating contribution to the correlation curve (Fig. $4 \mathrm{f}$ in [18]). If these bright peaks are rare events, they can be excluded from the analysis by recording a series of shorter FCS measurements instead of one long measurement and excluding the affected correlation curves from the calculation of an average FCS curve.

\subsection{Limitations of autocorrelation analysis for in vivo binding experiments}

A number of binding experiments have been performed in vitro, based on the change in diffusion time [31-35]. However, this kind of measurement is limited to cases with a considerable change in molecular mass on binding (binding of a small fluorescent ligand to a larger macromolecule) since the diffusion time scales only with the third root of the molecular mass. Also, the multitude of potential interactions in live cells and the inhomogeneity of the intracelllular environment probably preclude the in vivo use of this kind of binding analysis in most applications.

\section{Binding studies by dual-color cross-correlation}

An important extension of the fluorescence correlation technique described so far is provided by dual-color cross-correlation spectroscopy [36,37]. Here, two spectrally well-separated fluorophores (e.g., Alexa 488 and Cy5) are used to label two interacting molecules of interest in the sample and the fluorescence emissions from the two dyes are separated by means of a secondary dichroic mirror. The fluorescence signals are directed onto two detectors, i.e., the green and the red channels (Figs. 1, 6). Just as before, for each channel, the fluorescence autocorrelation curve according to Eq. (1) is calculated, providing concentration and mobility information for the red and the green particles. Additionally, the crosscorrelation between the fluorescence signals in the two channels is computed, yielding a third correlation curve: 


$$
\begin{aligned}
G_{x}^{F}(\tau) & =\left\langle F_{\text {red }}(t) F_{\text {green }}(t+\tau)\right\rangle /\left\langle F_{\text {red }}(t)\right\rangle\left\langle F_{\text {green }}(t)\right\rangle \\
\text { or } & \\
G_{x}^{\delta F}(\tau) & =\left\langle\delta F_{\text {red }}(t) \delta F_{\text {green }}(t+\tau)\right\rangle /\left\langle F_{\text {red }}(t)\right\rangle\left\langle F_{\text {green }}(t)\right\rangle \\
& =G_{\mathrm{x}}^{F}(\tau)-1 .
\end{aligned}
$$

In an idealized setup, the amplitude of the crosscorrelation curve is zero (or 1 , if the first definition is used), when only single-labeled particles are present, and it approaches the amplitudes of the autocorrelation curves for the special case of $100 \%$ binding, i.e., when all diffusing particles induce simultaneous, fully correlated fluctuations in both channels. In contrast to higher absolute amplitudes of the green and the red autocorrelation curves that indicate lower overall numbers of green and red labeled particles, a higher crosscorrelation amplitude relative to the autocorrelation amplitudes reflects a greater fraction of particles carrying both labels (Fig. 6). Therefore, cross-correlation analysis is well suited to the analysis of binding [38] of two differently labeled molecules, independent of any changes in diffusion time. The brightnesses of the red and the green particles in their respective channels do not need to be identical.

\section{Artifact risks in cross-correlation spectroscopy}

\subsection{Crosstalk}

With cross-correlation having clear advantages or even being the only choice for some applications, the drawback compared with autocorrelation is that the optical alignment of the experimental setup is much more elaborate, since detection volume overlap must be achieved, and there is the risk of artifacts if the possibility of crosstalk is not properly considered. To reduce crosstalk, i.e., the representation of the green dye in the red channel leading to artifactual cross-correlation, the emissions of the two dyes should be spectrally as far apart as possible. Even with spectrally well-separated dyes, crosstalk can lead to artifacts, if there are many more green particles compared with red particles or if the green particles are much brighter than the red particles. In the extreme case, when the fraction of red molecules and their relative brightness approach zero, the crosstalk amplitude approaches 100\% of the autocorrelation amplitudes in an ideally aligned setup (Fig. 7). An easy inherent check for crosstalk is provided by the correlation pattern of the fast dynamics (blinking) that many dyes exhibit, e.g., due to triplet dynamics. Whereas the blinking shoulder in the FCS curve of the green dye also appears in the crosstalk-induced cross-correlation curve (Fig. 7), real cross-correlation does not show this blinking shoulder, since the emission dynamics of the simply codiffusing green and red chromophores are fully uncorrelated (Fig. 8).

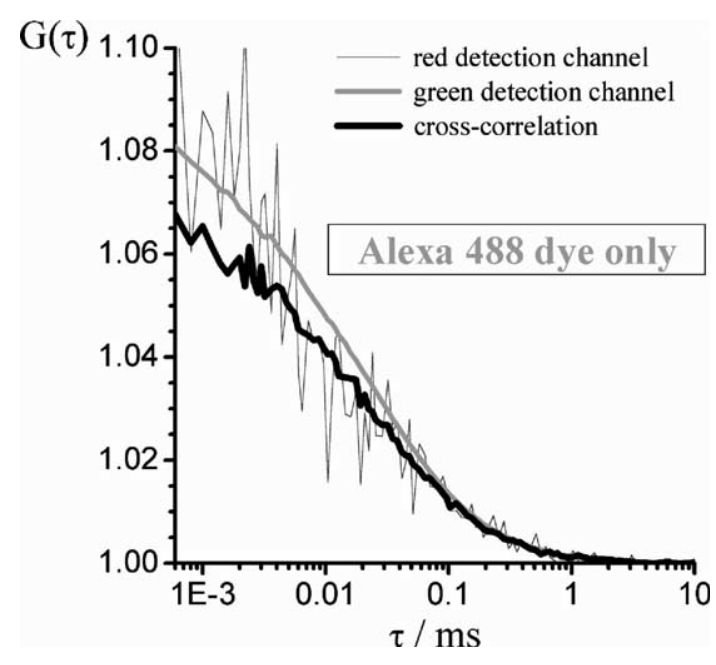

Fig. 7. Crosstalk. The fluorescence emission of the dye that is supposed to be detected in the green channel also extends into the transmission range of the filters in the red channel. If the detection volumes are well superimposed, this leads to ideally $100 \%$ crosstalk, although the particle brightness is low in the red channel. The crosstalk is reduced when there are enough real (i.e., bright) red particles present, so cross-correlation measurements are feasible despite crosstalk.

\subsection{Incomplete detection volume overlap}

To obtain maximum cross-correlation in two detection channels from double-labeled particles, it is imperative that the detection volumes overlap, as there is a certain probability of the molecules passing only one of the volumes and, thus, contributing to only the denominator of Eq. (5). As a consequence, cross-correlation curves computed from detection volumes that have a spatial offset $[39,40]$ suffer from a decreased amplitude. In addition, there is a change in the shape of the crosscorrelation curve, which for small offsets can be approximated by an apparently increased diffusion time in the cross-correlation curve compared with the autocorrelation curves [38]. For larger offsets, the maximum cross-correlation amplitude can be observed at time values $\tau_{\max }>0$, corresponding to the transit time between the two geometric centers of the volumes. For this case, the theory of spatial cross-correlation analysis strictly needs to be applied $[39,41]$. Therefore the detection volumes should be as coincident as possible. In the axial direction, this is complicated by the fact that for spectrally well-separated dyes, nonnegligible chromatic aberrations of the objective lenses play a decisive role, leading to imperfect axial overlap of the excitation profiles. In setups employing two pinholes (like the ConfoCor2), detection volume overlap can to some extent be improved by adjusting both detection beam paths (pinholes) to the same excitation volume (typically, the blue-shifted line) rather than their respective separated illumination volumes. Of course, this has the disadvantage that the red detection profile may be somewhat distorted and the signal in the red channel 

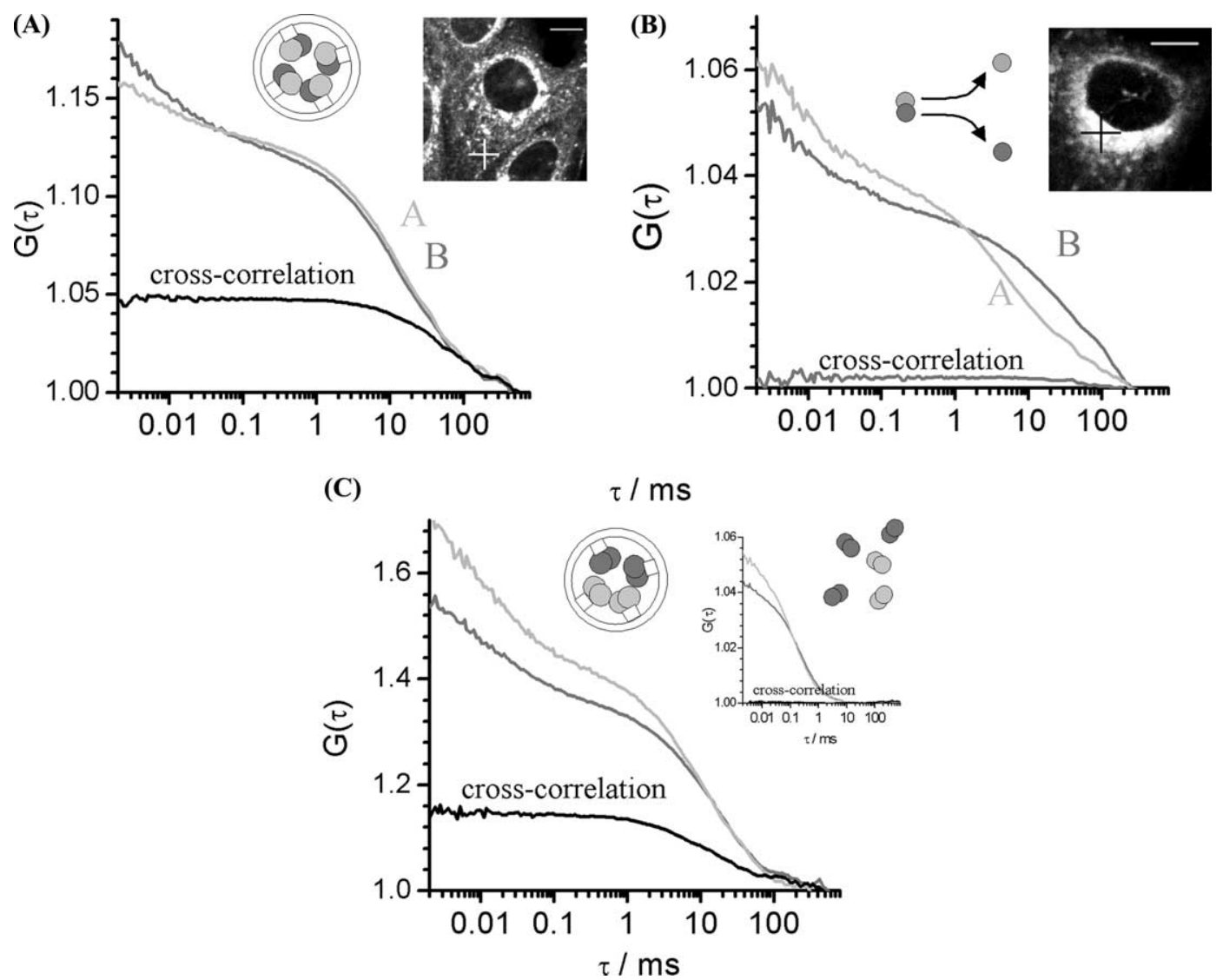

Fig. 8. Assessing endocytosis by dual-color cross-correlation spectroscopy [18]. (A) Inset: LSM image (bar $=10 \mu \mathrm{m}$ ) taken during the endocytosis of double-labeled cholera toxin by Vero cells, where the crosshair indicates the position of the FCS measurement: There is strong cross-correlation to be observed between the Cy2-labeled A subunit and the Cy5-labeled B subunit of the cholera toxin, since the two subunits are contained in the same small diffusing endocytic vesicles. (B) FCS curves obtained when the cholera toxin has reached the Golgi apparatus (LSM image: crosshair denotes FCS position; bar $=10 \mathrm{~m}$ ). After the immobile fraction has been bleached (not shown), the mobile fraction is analyzed: The low cross-correlation amplitude and the differing diffusion characteristics in the two autocorrelation channels indicate that A and B subunits have separated. (C) Inset: As opposed to double-labeled cholera toxin (A subunit labeled with Cy2, B subunit with Cy5), which already cross-correlates in solution (not shown), a mixture of Cy2-labeled holotoxin and Cy5-labeled holotoxin molecules does not cross-correlate in vitro. However, in the endocytic process, the differently labeled molecules colocalize in the same endocytic vesicles, leading to a strong cross-correlation signal (C).

reduced. A recommendable protocol for adjusting the pinholes on a ConfoCor 2 setup, where the excitations have been aligned by the manufacturer, makes use either of crosstalk of the green dye into the red channel or of the nonzero absorption of the red dye at short wavelengths: A concentrated solution of Alexa 488 or Cy5 is used, and both pinholes are adjusted using only the 488$\mathrm{nm}$ excitation. For de novo alignment in self-built setups, both pinholes are also adjusted to one excitation. Then, without changing the detection optics, the first excitation beam is blocked and the second beam is aligned to yield the same correlation curve in the red channel as before. The problem of positioning the excitation beams can be easily eliminated (except for the contribution of chromatic abberations) if a laser in multiline mode [42] or two-photon excitation of two dyes with the same IR wavelength is employed [29].

Apart from the spatial positioning of the focal volumes, there is the problem of different volume sizes due to the wavelength dependence of the diffraction limit. If the red volume is bigger than the green volume, the red autocorrelation curve has a lower amplitude than the green one. In this case, the cross-correlation amplitude can in first approximation, even for 100\% binding, only reach the amplitude of the lower (red) autocorrelation. A possibility is to adjust the size of the red excitation volume by controlling the overfilling of the back aperture of the objective with a telescope system [36]. In setups with adjustable pinholes, the detection volumes can to some extent be regulated through the pinhole sizes.

\subsection{Photobleaching}

Another artifact source in cross-correlation is photobleaching, which is normally easy to recognize. A strong decay in the count rate due to photobleaching that appears simultaneously in both channels will give 
rise to a cross-correlation amplitude. Therefore, as in fluorescence autocorrelation spectroscopy, strong photobleaching has to be avoided. In the case of particles that are immobile on the FCS time scale, one can resort to the cross-correlation of position-dependent instead of time-dependent signals (colocalization analysis by image correlation spectroscopy $[43,44])$. Another possibility is to convert the position-dependent signal into a time-dependent signal by scanning the FCS volume [3].

\section{In vivo applications of dual-color cross-correlation spectroscopy}

One of the difficulties in performing dual-color crosscorrelation spectroscopy in vivo is that as mentioned above, among the fluorescent proteins that are currently available there seems to be no ideal combination of two proteins that fulfill all necessary criteria: good brightness and photostability parameters, low tendencies of aggregation, and small spectral overlap. Therefore, if intracellular dual-color cross-correlation is to be performed, at least one of the target molecules needs to be labeled externally and be introduced into the cell using methods involving loading reagents ("protein transfection"), electroporation, or microinjection. Naturally, this problem does not arise when studying endocytic processes by FCS. It has recently been shown [18] that it is possible to follow the fate of the A and B subunits of cholera toxin on endocytosis by single-photon dualcolor cross-correlation spectroscopy, using the commercial ConfoCor2 setup (Fig. 8). The A subunit of cholera toxin was labeled with the green fluorophore $\mathrm{Cy} 2$ and the B subunit with the red fluorophore Cy5. The holotoxin binds to the GM1 receptor at the plasma membrane, where FCS measurements could not be performed due to its too low mobility. When the holotoxin has been taken up into endosomal vesicles, however, strong cross-correlation between the A and B subunits can be observed, and diffusion times indicate that particles of the size of whole vesicles (not single proteins) diffuse through the focus. Thus, A and B subunits are in the same small diffusing compartment. When the toxin reaches the Golgi, there is an immobile, possibly membrane-bound fraction and a mobile fraction. The latter fraction shows a significantly reduced relative cross-correlation amplitude and differing diffusion characteristics of the two subunits. Both observations indicate that the A and B subunits have adopted individual pathways. Along these lines, dual-color crosscorrelation was able to confirm the results on live cells that had previously been obtained by FRET on fixed cells.

The observation of the motion of complete vesicles containing the toxin molecules has some interesting as- pects. First, the diffusing particles are very bright as the vesicles contain several fluorophores, which leads to very low noise in the FCS curves. Second, since crosscorrelation arises from cargo colocalization within the vesicles, the method should be applicable to the investigation of joint usage of the same endocytic pathway by different cargos. FRET, being the only other technique that has so far been used to analyze molecular association processes in live cells, is not a valuable alternative for this purpose, since cargo molecules in a vesicle are not necessarily close enough on a molecular scale. Static colocalization analysis from confocal images, frequently employed by users of standard scanning microscopy units, is also a less suitable method, because it primarily picks up fluorescence signals deriving from larger structures. In addition, it does not make use of concomitant movement as required for fluorescence crosscorrelation spectroscopy, the latter thus being a much stricter criterion for a true physical or chemical connection or linkage between particles, since particles being incidentally on the same spot at a certain time would not substantially contribute to the coanalysis. The principle of this application of cross-correlation has been demonstrated by having cells endocytose a mixture of differently single-labeled cholera toxin molecules, which do not show cross-correlation in buffer solution, but do show cross-correlation from endocytic vesicles in the cytoplasm (Fig. 8), because they use the same endocytic pathway.

As laid out in Section 1, the ultimate goal is to apply fluorescence cross-correlation spectroscopy to monitor reversible binding directly in the live cell. This has recently been accomplished for the first time by Kim et al. [45] using two-photon fluorescence cross-correlation spectroscopy. They succeeded in following the binding and unbinding of Alexa 633-labeled calmodulin and eGFP-labeled calcium-calmodulin-dependent protein kinase II (CaMKII) on changes in calcium level in live cells.

\section{Acknowledgments}

We thank Sally Kim for unpublished data on CaMKII, Dag Scherfeld for collaborating on the GUVs, and other members of the Experimental Biophysics Group for discussions.

\section{References}

[1] R. Virchow, Die Cellularpathologie (1858).

[2] A. Gennerich, D. Schild, Biophys. J. 83 (2002) 510-522.

[3] A. Amediek, E. Haustein, D. Scherfeld, P. Schwille, Single Mol. 3 (2002) 201-210.

[4] D. Magde, E.L. Elson, W.W. Webb, Phys. Rev. Lett. 29 (1972) 705-708. 
[5] E.L. Elson, D. Magde, Biopolymers 13 (1974) 1-27.

[6] D. Magde, E.L. Elson, W.W. Webb, Biopolymers 13 (1974) 29-61.

[7] R. Rigler, J. Widengren, Bioscience 3 (1990) 180-183.

[8] R. Rigler, U. Mets, J. Widengren, P. Kask, Eur. Biophys. J. 22 (1993) 169-175.

[9] Y. Chen, J.D. Muller, P.T.C. So, E. Gratton, Biophys. J. 77 (1999) 553-567.

[10] J.D. Muller, Y. Chen, E. Gratton, Biophys. J. 78 (2002) 474-486.

[11] Y. Chen, J.D. Muller, Q.Q. Ruan, E. Gratton, Biophys. J. 82 (2002) 133-144.

[12] P. Kask, K. Palo, N. Fay, L. Brand, U. Mets, D. Ullmann, J. Jungmann, J. Pschorr, K. Gall, Biophys. J. 78 (2000) 17031713.

[13] N.L. Thompson, in: J.R. Lakowicz (Ed.), Topics in Fluorescence Spectroscopy, vol. 1, Plenum, New York, 1991, pp. 337-378.

[14] O. Krichevsky, G. Bonnet, Rep. Prog. Phys. 65 (2002) 251-297.

[15] J. Widengren, U. Mets, R. Rigler, J. Phys. Chem. 99 (1995) 13368-13379.

[16] J. Widengren, P. Schwille, J. Phys. Chem. A 104 (2000) 64166428.

[17] U. Haupts, S. Maiti, P. Schwille, W.W. Webb, Proc. Natl. Acad. Sci. USA 95 (1998) 13573-13578.

[18] K. Bacia, I.V. Majoul, P. Schwille, Biophys J. 83 (2002) 1184 1193

[19] P.W. Wiseman, J.A. Squier, M.H. Ellisman, K.R. Wilson, J. Microsc. Oxford 200 (2000) 14-25.

[20] P. Dittrich, F. Malvezzi-Campeggi, M. Jahnz, P. Schwille, Biol. Chem. 382 (2001) 491-494.

[21] R.Y. Tsien, Annu. Rev. Biochem. 67 (1998) 509-544.

[22] P. Schwille, S. Kummer, A.A. Heikal, W.E. Moerner, W.W. Webb, Proc. Natl. Acad. Sci. USA 97 (2000) 151-156.

[23] F. Malvezzi-Campeggi, M. Jahnz, K.G. Heinze, P. Dittrich, P. Schwille, Biophys. J. 81 (2001) 1776-1785.

[24] T. Kohl, K.G. Heinze, R. Kuhlemann, A. Koltermann, P. Schwille, Proc. Natl. Acad. Sci. USA 99 (2002) 12161-12166.

[25] R.E. Campbell, O. Tour, A.E. Palmer, P.A. Steinbach, G.S. Baird, D.A. Zacharias, R.Y. Tsien, Proc. Natl. Acad. Sci. USA 99 (2002) 7877-7882.

[26] W. Denk, J.H. Strickler, W.W. Webb, Science 248 (1990) 73-76.
[27] W. Denk, D.W. Piston, W.W. Webb, in: J.B. Pawley (Ed.), Handbook of Biological Confocal Microscopy, Plenum, New York, 1995, p. 445ff.

[28] P. Schwille, U. Haupts, S. Maiti, W.W. Webb, Biophys. J. 77 (1999) 2251-2265.

[29] K.G. Heinze, A. Koltermann, P. Schwille, Proc. Natl. Acad. Sci. USA 97 (2000) 10377-10382.

[30] U. Meseth, T. Wohland, R. Rigler, H. Vogel, Biophys. J. 76 (1999) 1619-1631.

[31] M. Kinjo, R. Rigler, Nucleic Acids Res. 23 (1995) 1795-1799.

[32] B. Rauer, E. Neumann, J. Widengren, R. Rigler, Biophys. Chem. 58 (1996) 3-12.

[33] P. Schwille, F. Oehlenschläger, N.G. Walter, Biochemistry 35 (1996) 10182-10193.

[34] J. Schuler, J. Frank, U. Trier, M. Schafer-Korting, W.A. Saenger, Biochemistry 38 (1999) 8402-8408.

[35] T. Wohland, K. Friedrich, R. Hovius, H. Vogel, Biochemistry 38 (1999) 8671-8681.

[36] P. Schwille, F.J. Meyer-Almes, R. Rigler, Biophys. J. 72 (1997) 1878-1886.

[37] P. Schwille, in: R. Rigler, E.S. Elson (Eds.), Fluorescence Correlation Spectroscopy: Theory and Applications, Springer, Berlin, 2001, pp. 360-378.

[38] T. Weidemann, M. Wachsmuth, M. Tewes, K. Rippe, J. Langowski, Single Mol. 3 (2002) 49-61.

[39] M. Brinkmeier, K. Dorre, J. Stephan, M. Eigen, Anal. Chem. 71 (1999) 609-616.

[40] M. Brinkmeier, in: R. Rigler, E.S. Elson (Eds.), Fluorescence Correlation Spectroscopy: Theory and Applications, Springer, Berlin, 2001, pp. 379-395.

[41] P. Dittrich, P. Schwille, Anal. Chem. 74 (2002) 4472-4479.

[42] T. Winkler, U. Kettling, A. Koltermann, M. Eigen, Proc. Natl. Acad. Sci. USA 96 (1999) 1375-1378.

[43] N.O. Petersen, in: R. Rigler, E.S. Elson (Eds.), Flourescence Correlation Spectroscopy: Theory and Applications, Springer, Berlin, 2001, pp. 162-184.

[44] C.M. Brown, M.G. Roth, Y.I. Henis, N.O. Petersen, Biochemistry 38 (1999) 15166-15173.

[45] S. Kim, K.G. Heinze, M.N. Waxham, P. Schwille, Biophys. J. (Annual Meeting Abstracts) 2002. 